Editorial

\title{
Fracture and damage analysis in design
}

This Special Issue contains selected papers presented at the XXIX National Conference of the Italian Association for Stress Analysis (AIAS), held in Maratea, organized by University of Calabria, (Italy) from 7 to 10 September 2010.

This Conference is focussed on several topics, the common matrix being always the (experimental but also numerical and theoretical) analysis of the state of stress.

The attendants to the Conference, which is annual, are always numerous: their works represent the state of art of the research in the topic of Stress Analysis in Italy.

The Conference had different sessions, among these latter the ones related to fracture and damage attracted many authors and participants. A part of the papers presented in these sessions (by considering in particular the design application) was selected by the Scientific Committee of the Conference to be submitted for publication on this Special Issue and, after a peer review process that included many international referees, some of them are now published.

We thank both the authors and the referees for their remarkable and patient work.

The selected works cover both experimental and computational aspects, but are mainly devoted to the application of fracture and damage mechanics and of fatigue life prediction criteria to engineering practical application. This fact reflects very well the aim and the activity of the Association, which would like to consider the Universities competences and the industry technical problems. We hope that the papers selected will be of interest also for the readers of Engineering Fracture Mechanics.

Last but not least, a special thank is due, once again, to Professor Karl-Heinz Schwalbe for giving us the opportunity to publish this Special Issue and for his patient and continuous help throughout this project.

Laura Vergani

Dipartimento di Meccanica, Politecnico di Milano, Milan, Italy

Renzo Capitani

Dipartimento di Meccanica e Tecnologie Industriali, Università degli Studi di Firenze, Florence, Italy 